\title{
Solution of Burger's Equations by Orthogonal Collocation on Finite Elements Hermite Basis
}

\author{
Dr. A K Mittal ${ }^{1}$, and Dr. V K Kukreja ${ }^{2}$
}

\begin{abstract}
Nonlinear parabolic Burgers' equations arising in unsteady flow of the generalized Newtonian fluid are solved numerically by Orthogonal Collocation on Finite Elements using Hermite Basis. The technique is used for discretization along with the zeros of Chebyshev polynomial as collocation points. Results are compared with exact solution and error analysis is shown for different techniques used earlier for the solution of the Burger's equations.
\end{abstract}

Keywords - Burgers' Equation, Chebyshev Polynomial, OCFE.

\section{INTRODUCTION}

$\mathrm{C}$

$U_{t}+U U_{x}=\lambda U_{x x}, a \leq x \leq b, t \geq 0$,

with the initial condition

$$
U(x, 0)=f(x), a \leq x \leq b,
$$

and the boundary condition

$$
U(a, t)=\beta_{1}, \quad U(b, t)=\beta_{2},
$$

where $\lambda>0$ is the coefficient of kinematics viscosity, and $\beta_{1}, \beta_{2}$ and $f(x)$ will be chosen in a later section. This is a nonlinear parabolic equation [1] and describes in a simple manner a balance between nonlinear convection and linear diffusion or dissipation.

Burgers' equation is well-known to show shock formation. It is the 1D Navier-Stokes equation without the pressure term and the volume forces. Due to its similarity to the NavierStokes equation, Burgers' equation often arises in the mathematical modelling used to solve problems in fluid dynamics involving turbulence. Bateman [2] has first introduced Burgers' equation as worthy of study and gave its steady solutions. After that Burgers [3] simplified the NavierStokes equation by just dropping the pressure term. It was later treated as a mathematical model for turbulence and such an equation is widely referred to as Burgers' equation. Since then the equation has found applications in field as diverse as number theory, gas dynamics, heat conduction, elasticity, etc. However, Hopf [4] and Cole [5] have shown that the homogeneous Burgers' equation lacks the most important

Ajay Mittal ${ }^{1}$ is with the Department of Mathematics, Aryabhatta Group of Institutes, Barnala, Punjab 148101 INDIA.

V Kukreja ${ }^{2}$ is with Department of Mathematics, Sant Longowal Institute of Engineering \& Technology, Longowal, Punjab 148101 INDIA. property attributed to turbulence. The exact solutions of the one dimensional Burgers' equation have been surveyed by Benton and Platzman [6]. In many cases these solutions involve infinite series which may converge very slowly for small values of the viscosity coefficient $\lambda$ which correspond to steep wave fronts in the propagation of the dynamic waveforms. Many studies have been done on the numerical solutions of Burgers' equation to deal with solutions for the small values of $\lambda$. A finite element method has been given by Caldwell et. al. [7], to solve Burgers' equation by altering the size of the element at each stage. Moreover Caldwell and Smith [8] have discussed the comparison of a number of numerical approaches to the equation. Nguyen and Reynen [9] also suggested a space-time finite element method based on a least square weak formulation using piecewise linear shape functions. A kind of finite element method based on weighted residual formulation is given by Varo glu and Liam Finn [10] and demonstrated the high accuracy and the stability.

In the present work, the model equations are discretized using the technique of orthogonal collocation on finite elements with hermite basis. OCFE is used for approximating function, with zeros of shifted Chebyshev polynomials as collocation points. Solution and its principal derivatives over the subinterval are approximated. Placing nodal values and its derivatives in the Burgers' equation are resulting in system consisting of $n+1$ equations for $n+3$ parameters. The resulting system can be solved with MATLAB after the boundary conditions are applied.

\section{NUMERICAL SCHEME}

\section{A. OCFE Using Hermite Basis}

Hermite cubic interpolant of function $f$ relative to partition $a=x_{0}<x_{1}<\ldots<x_{n}=b$ is a function $s$ that satisfies:

- $s$ coincides with a cubic polynomial $s_{j}(x)$, on each subinterval $\left[x_{j}, x_{j+1}\right]$, where $j=0,1,2, \ldots, n-1$,

- $s$ interpolates $f$ and $f^{\prime}$ at $x_{0}, x_{1}, \ldots, x_{n}$,

- $s$ and $s^{\prime}$ are continues on $[a, b]$.

The Hermite cubic Interpolation of $f$ and its first derivative at $x=x_{j}$ requires that 
$s_{j}\left(x_{j}\right)=f\left(x_{j}\right)$ and $s_{j}^{\prime}\left(x_{j}\right)=f^{\prime}\left(x_{j}\right)$.

At $x=x_{j+1}$, combining the continuity of $s$ and $s^{\prime}$ with interpolation of $f$ and $f^{\prime}$ one gets:

$s_{j}\left(x_{j+1}\right)=s_{j+1}\left(x_{j+1}\right)=f\left(x_{j+1}\right)$ and

$s_{j}^{\prime}\left(x_{j+1}\right)=s_{j+1}^{\prime}\left(x_{j+1}\right)=f^{\prime}\left(x_{j+1}\right)$.

$s_{j}(x)$ is a third degree polynomial that interpolates both

$f$ and $f^{\prime}$ at $x=x_{j}$ and at $x=x_{j+1}$.

Therefore:

$$
\begin{gathered}
s_{j}(x)=H_{1, j}(x) f\left(x_{j}\right)+H_{1, j+1}(x) f\left(x_{j+1}\right) \\
+\hat{H}_{i, j}(x) f^{\prime}\left(x_{j}\right)+\hat{H}_{i, j+1}(x) f^{\prime}\left(x_{j+1}\right) \\
\text { where, } H_{1, j}(x)=\left[1-2 \frac{x-x_{j}}{x_{j}-x_{j+1}}\right]\left(\frac{x-x_{j+1}}{x_{j}-x_{j+1}}\right)^{2}, \\
H_{1, j+1}(x)=\left[1-2 \frac{x-x_{j+1}}{x_{j+1}-x_{j}}\right]\left(\frac{x-x_{j}}{x_{j+1}-x_{j}}\right)^{2}, \\
\hat{H}_{i, j}(x)=\left(x-x_{j}\right)\left(\frac{x-x_{j+1}}{x_{j}-x_{j+1}}\right)^{2}, \\
\hat{H}_{i, j+1}(x)=\left(x-x_{j+1}\right)\left(\frac{x-x_{j}}{x_{j+1}-x_{j}}\right)^{2} .
\end{gathered}
$$

In the method of orthogonal collocation on finite elements using Hermite basis, the axial domain $(0 \leq x \leq 1)$ is divided into small sub domains called 'elements'. The global variable $x$ varies in the $\ell^{\text {th }}$ element, where $\ell=1,2, \ldots, n e$. The node points are set at $x_{1}, x_{2}, \ldots, x_{n e+1}$ as shown in Fig 1 . The boundary points 0 and 1 have been placed at $x_{1}=0$ and $x_{n e+1}=1$. To apply the orthogonal collocation with in $\ell^{\text {th }}$ element, a variable $u=\frac{x-x_{\ell}}{x_{\ell+1}-x_{\ell}}$ is introduced in $\ell^{\text {th }}$ element in such a way that as $x$ varies from $x_{\ell}$ to $x_{\ell+1}, u$ varies from 0 to 1 , as shown in Fig 2 . Then the orthogonal collocation is applied within each element. The Hermite polynomials are $C^{1}$ continuous; therefore the trial function and its first derivative are automatically continuous at the nodal points or at the boundaries of the elements.

\section{B. Collocation Points}

Choice of the collocation points is an important and sensitive part of the orthogonal collocation method. In general, collocation points are taken to be the zeros of orthogonal polynomials like Legendre polynomial or Chebyshev polynomial. These polynomials are particular case of Jacobi polynomials, given as:

$$
\begin{aligned}
P_{n}^{(\alpha, \beta)}(z)= & \frac{\Gamma(n+\alpha+1) \Gamma(n+\beta+1)}{n ! \Gamma(n+\alpha+\beta+1)} \\
& \sum_{m=0}^{n}\left(\begin{array}{l}
n \\
m
\end{array}\right) \frac{\Gamma(\alpha+\beta+n+m+1)}{\Gamma(\alpha+m+1)}\left(\frac{z-1}{2}\right)^{m}
\end{aligned}
$$

The Legendre and Chebyshev polynomials are special cases of Jacobi polynomials for $\alpha=\beta=0$ and $\alpha=\beta=1 / 2$, respectively. In axial domain, the zeros of shifted Legendre polynomial have been taken as collocation points. The zeros of Legendre polynomial are calculated from the following recurrence relation:

$$
\begin{aligned}
(j-1) P_{j-1}(x)= & (2 j-3) x P_{j-2}(x) \\
& -(j-2) P_{j-3}(x)
\end{aligned} ; j=2, \ldots, m+1,
$$

where $P_{0}(x)=1$ and $P_{-1}(x)=0$. In case of Legendre polynomial, 0 and 1 are taken to be the boundary points. The collocation points are obtained by mapping the computational domain of the interval $[-1,1]$ to $[0,1]$ with the help of following relationship:

$u_{m+3-j}=\frac{x_{j}}{2}+\frac{1}{2}$

where $x_{j}$ is the $j^{\text {th }}$ collocation point in the interval $[-1,1]$.

Chebyshev polynomial has the tendency to keep the error down to a minimum at the corners [11]. Therefore, the zeros of shifted Chebyshev polynomial have been used as collocation points in the radial domain, because the results are required at the corner in radial domain.

The $m+2$ interpolation points are chosen to be the extreme values of an $(m+1)^{\text {th }}$ order shifted Chebyshev polynomial:

$x_{j}=\cos \frac{\pi(j-1)}{m+1} ; j=1,2, \ldots, m+2$.

The discretization end points are fixed as 0 and 1 .

\section{C.Discretization Using Approximating Function}

The dimensionless form of the model equations along with boundary and initial conditions are discretized using the approximating function.

The approximate solution $c(u, t)$ at the $j^{\text {th }}$ collocation point in the $i^{\text {th }}$ element is given by:

$$
\begin{aligned}
c_{i j}(u, t)=\sum_{l=1}^{4} a_{l+2(i-1)}^{i j}(t) H_{l}^{i}(u) & \\
& \quad i=1,2, \ldots, k ; j=2,3 .
\end{aligned}
$$

where the four coefficients $a_{l+2(i-1)}^{i j}(t)$ in each element are unknown parameters. The labeling of the parameters are 
such that the solutions $c_{k}(u, t)$ and $c_{k+1}(u, t)$ are continuous at their common node points. The derivatives of trial function (3) can be written as follows:

$$
\frac{\partial c_{i j}}{\partial u}=\frac{1}{h_{i}} \sum_{l=1}^{4} a_{l+2(i-1)}^{i j} \frac{d H_{l}^{i}}{d u}
$$

(9)

$$
\begin{aligned}
& \frac{\partial^{2} c_{i j}}{\partial u^{2}}=\frac{1}{h_{i}^{2}} \sum_{l=1}^{4} a_{l+2(i-1)}^{i j} \frac{d^{2} H_{l}^{i}}{d u^{2}} \\
& \frac{\partial c_{i j}}{\partial t}=\sum_{l=1}^{4} \frac{d a_{l+2(i-1)}^{i j}}{d t} H_{l}^{i}
\end{aligned}
$$

The trial function is composed of value of the function and first derivative at each end in such a manner that three of these quantities are zero and fourth is one. In particular, for $k^{\text {th }}$ element $\left[x_{k}, x_{k+1}\right]$ cubic Hermite basis functions are given as follows:

$$
\begin{aligned}
& H_{1}^{k}(\xi)=(1-\xi)^{2}(1+2 \xi) \\
& H_{2}^{k}(\xi)=\xi(1-\xi)^{2} \\
& H_{3}^{k}(\xi)=\xi^{2}(\xi-1) \\
& H_{4}^{k}(\xi)=\xi^{2}(3-2 \xi)
\end{aligned}
$$

Detailed

\section{APPLICATION OF NUMERICAL APPROACH}

\section{A. Problem 1}

Consider the Burger's equation (1) with the initial condition:

$$
U(x, 0)=\sin \pi x, 0 \leq x \leq 1
$$

and the homogeneous boundary conditions:

$$
U(0, t)=U(1, t)=0, t>0
$$

The exact solution of Burger's equation (1) with conditions (12) and (13) was given by Cole [5] as:

$$
U(x, t)=2 \pi \lambda \frac{\sum_{n=1}^{\infty} b_{n} \exp \left(-n^{2} \pi^{2} \lambda t\right) n \sin n \pi x}{b_{0}+\sum_{n=1}^{\infty} b_{n} \exp \left(-n^{2} \pi^{2} \lambda t\right) n \cos n \pi x}
$$

where $b_{0}=\int_{0}^{1} \exp \left[-(2 \pi \lambda)^{-1}(1-\cos \pi x)\right] d x$ and $b_{n}=2 \int_{0}^{1} \exp \left[-(2 \pi \lambda)^{-1}(1-\cos \pi x)\right] \cos n \pi x d x, n \geq 1$ are Fourier coefficients.
The theoretical solution of this problem was expressed as an infinite series by Cole [5]. For different viscosity parameters, a comparison of results for present work with the previous work $[12,13,14,15]$ at $t=0.1$ for $v=1, \Delta t=$ $0.00001, h=0.0125$, are presented in Table 1. Error analysis comparison of results at $t=0.1$ for $\lambda=1, \Delta t=0.00001, h=$ 0.0125 is shown in Table 2. A comparison of present method is shown in Table 3, with previous ones reported by $[14,15]$. In Figure 3, the error analysis comparison of results at $t=0.1$ for $\lambda=1, \Delta t=0.00001, h=0.0125$ has presented.

\section{B. Problem 2}

The Eq.(1) with boundary conditions (13) and initial condition

$u(x, 0)=\frac{2 \pi \nu \sin \pi x}{b+\cos \pi x}, b>0$

The exact solution of this problem given by Wood [16]:

$$
u(x, t)=\frac{2 \pi v \exp \left(-\pi^{2} v t\right) \sin \pi x}{b+\exp \left(-\pi^{2} v t\right) \cos \pi x}, b>0
$$

In order to compare the solutions with [14], we choose the parameters $b=2, t=0.001, \Delta t=0.0001, h=0.025$, with different choice of viscosity are displayed in Table 4 and its observed that results obtained by OCFE for using different types of collocation points (i.e zeros of shifted Chebyshev polynomial and zeros of shifted Legendre polynomial) with $v$ $=0.004,0.005$ at different $x$ and $t$ are better and shows near matching with exact solution.

3D graph of problem 2 is exhibited in figure 4 which reveals that as viscosity gets smaller, a very sharp front near the left boundary and the steepness of the solution increases sharply near the right boundary. This steepness is controlled by taking small step for space variable $x$.

\section{CONCLUSION}

In this paper, the technique of orthogonal collocation on finite elements with Hermite basis. is presented to solve Burger's equation which illustrates the validity and accuracy of the given method. The OCFE with Hermite basis is inherently smoother than other methods available in literature. It has wide applicability to different engineering problems.

\section{REFERENCES}

[1] B. A. Finlayson, "Nonlinear Analysis in Chemical Engineering. New York: Mc Graw-Hill Inc. USA 1980, 1-314.

[2] H. Bateman, "Some recent researches on the motion of fluids", Monthly Weather Rev. 43, pp. 163-170, 1915. https://doi.org/10.1175/1520-0493(1915)43<163:SRROTM>2.0.CO;2

[3] J. M. Burger, "A Mathematical Model Illustrating the Theory of Turbulence", in Adv. in App. Mech. I, Academic Press, New York, pp. $171-199,1948$.

https://doi.org/10.1016/s0065-2156(08)70100-5

[4] E. Hopf, "The partial differential equation Ut p UUx 1/4 1Uxx, Comm. Pure App. Math. 3, pp. 201-230, 1950.

[5] J. D. Cole, "On a quasi-linear parabolic equations occurring in aerodynamics", Quart. Appl. Math. 9 pp. 225-236, 1951.

https://doi.org/10.1090/qam/42889 
[6] E. Benton and G. W. Platzman, "A table of solutions of the onedimensional Burgers' equations", Quart. Appl. Math. 30, pp. 195-212, 1972.

https://doi.org/10.1090/qam/306736

[7] J. Caldwell, P. Wanless and A. E. Cook, "A finite element approach to Burgers' equation, Appl. Math. Modelling 5, pp. 189-193, 1981. https://doi.org/10.1016/0307-904X(81)90043-3

[8] J. Caldwell and P. Smith, "Solution of Burgers' equation with large Reynold's number", Appl. Math. Modelling 6, pp. 381-385, 1982. https://doi.org/10.1016/S0307-904X(82)80102-9

[9] H. Nguyen and J. Reynen, "A space-time finite element approach to Burgers' equation", in E. Hinton et al. (Eds.), Numerical Methods for Nonlinear Problems, vol. 3, Pineridge Press, pp. 718-728, 1987.

[10] E. Varo_glu and W. D. Liam Finn, "Space-time finite elements incorporating characteristics for the Burgers' equation", Int. J. Num. Meth. Engng. 16, pp. 171-184, 1983.

[11] S. C. Chen, E. K. C Lee. and Y. I. Chang, "Effect of the coordination number of the pore network on the transport and deposition of particles in porous media", Sep. Purif. Technol. 30, pp. 11-26, 2003. https://doi.org/10.1016/S1383-5866(02)00096-5
[12] I. Dag, D. Irk and B. Saka, "A Numerical solution of the Burgers' equation using cubic B-splines", Appl. Math. Comput. 163, pp. 199211, 2005.

https://doi.org/10.1016/j.amc.2004.01.028

[13] S. Kutulay, A. Esen, I. Dağ, "Numerical solutions of the Burgers' equation by the least-squares quadratic B-spline finite element method", J. Comput. Appl. Math. 167, pp. 21-33, 2004. https://doi.org/10.1016/j.cam.2003.09.043

[14] A. Asaithambi, "Numerical solution of the Burgers' equation by automatic differentiation", Appl. Math. Comput. 216, pp. 2700-2708, 2010 .

https://doi.org/10.1016/j.amc.2010.03.115

[15] M. Xu, R. H. Wang, J. H. Zhang and Q. Fang, "A novel numerical scheme for solving Burgers' equation”, Appl. Math. Comput. 217, pp. 4473-4482, 2011.

https://doi.org/10.1016/j.amc.2010.10.050

[16] W. L. Wood, "An exact solution for Burger's equation", Commun. Numer. Meth. Eng. 22, pp. 797-79, 2006. https://doi.org/10.1002/cnm.850

\section{FIGURES}

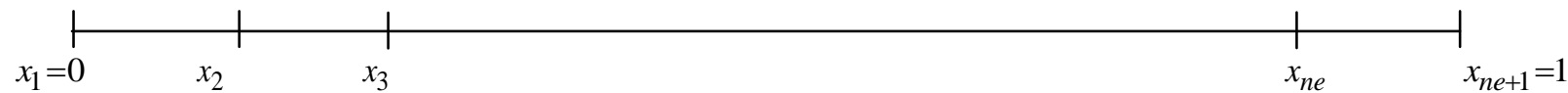

Fig. 1 Finite elements on global domain

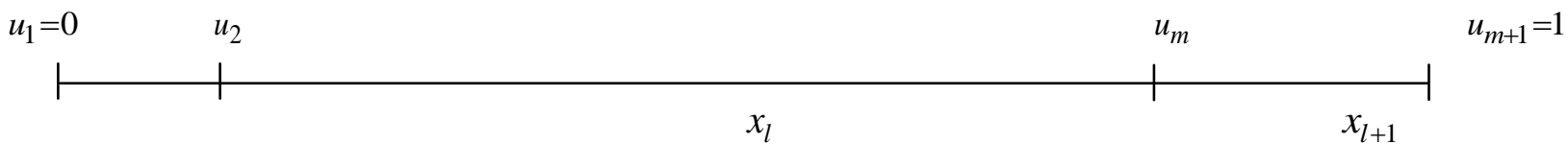

Fig. 2 Orthogonal collocation on local domain

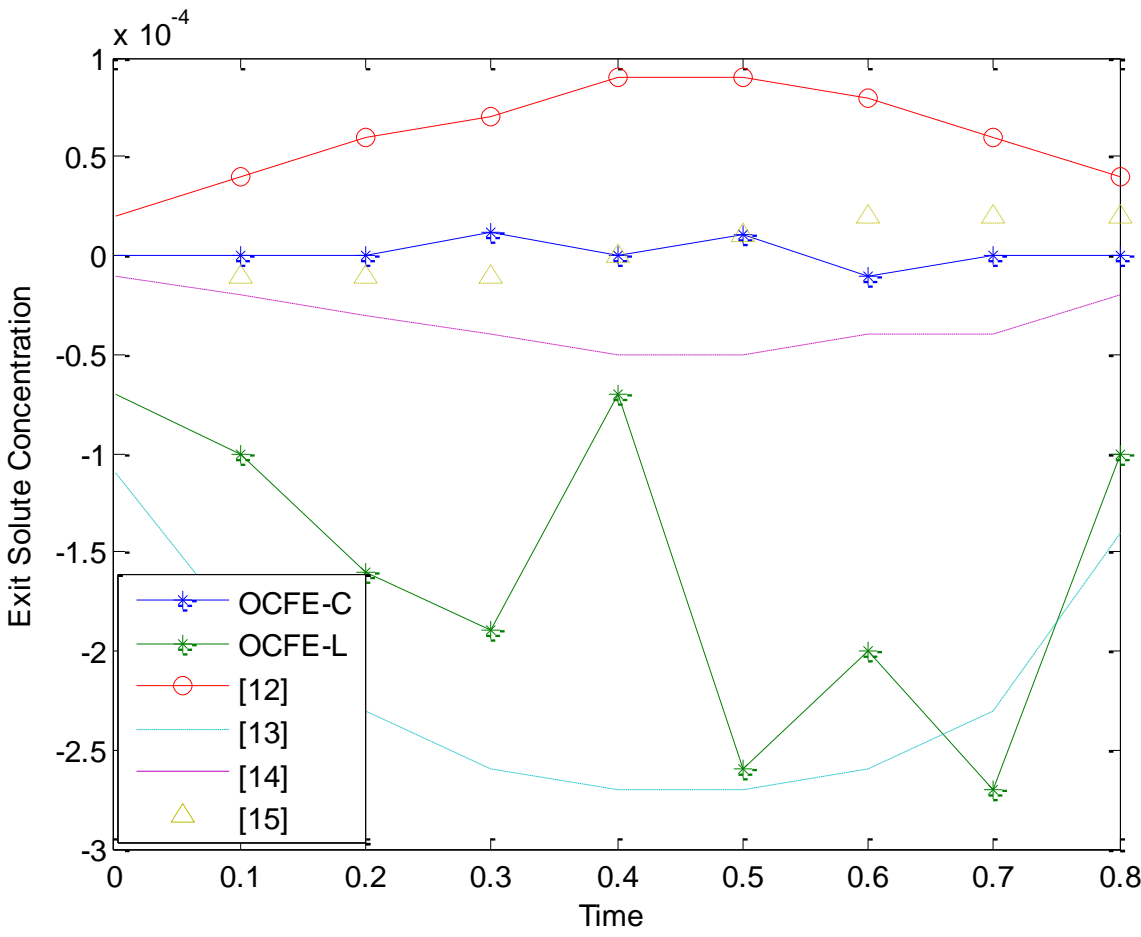

Fig. 3 Error analysis for Problem 1 at $t=0.1$ for $\lambda=1, \Delta t=0.00001, h=0.0125$. 

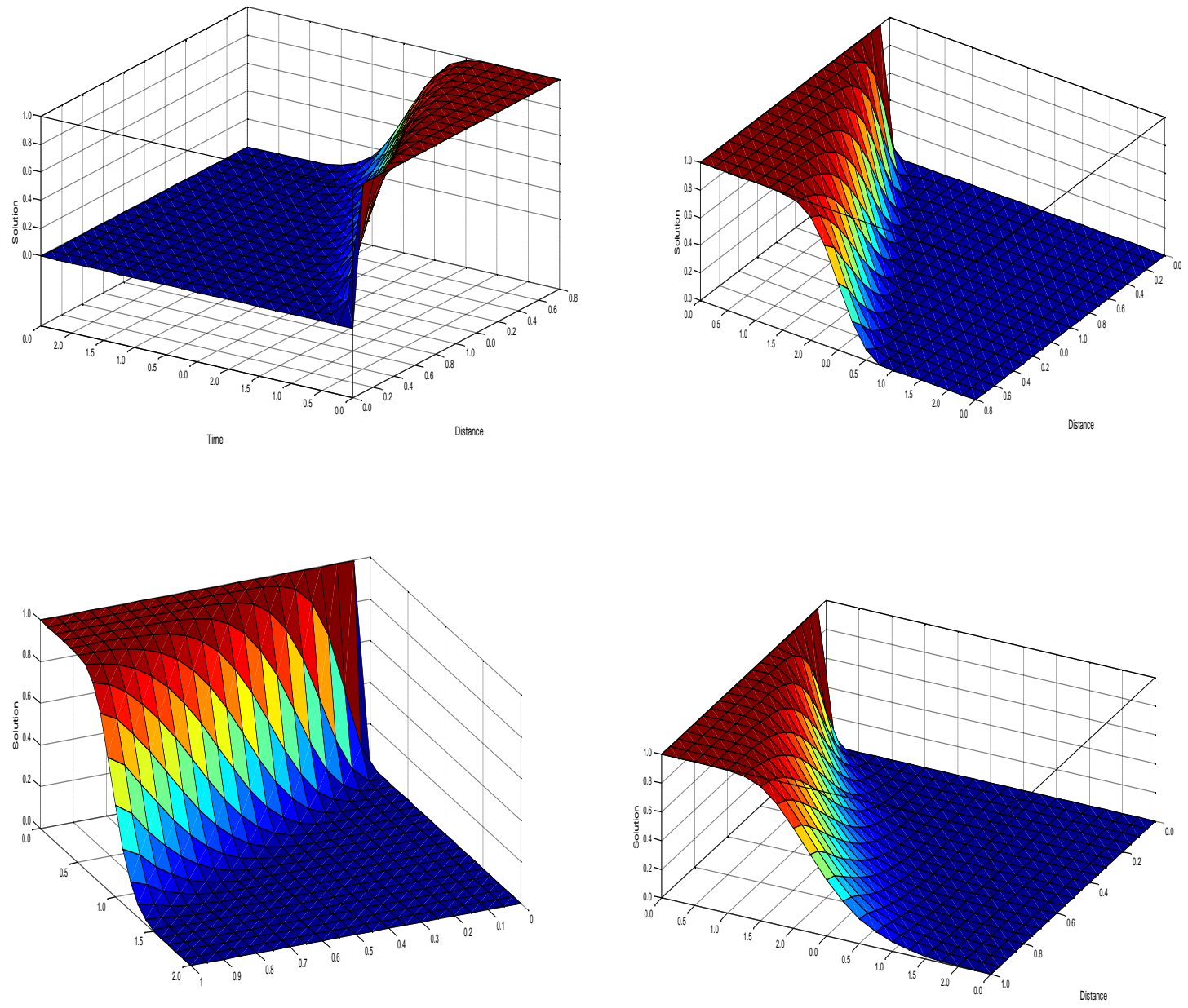

Fig. 4 Physical behavior of numerical solutions of Problem 2 for $h=0.0125$ and different values of $v$ and $\Delta t$ (a) $v=1, \Delta t=0.0001$ (b) $v=0.01, \Delta t=0.0001$ (c) $v=0.004, \Delta t=0.0001$ (d) $v=0.005, \Delta t=0.0001$

TABLES

TABLE I

COMPARISON OF RESULTS AT $T=0.1$ FOR $N=1, \Delta T=0.00001, H=0.0125$.

\begin{tabular}{|c|c|c|c|c|c|c|c|c|}
\hline$X$ & Exact & OCFE-L & OCFE-C & [12] & [13] & EFDM[14] & EEFDM[14] & [15] \\
\hline 0.1 & 0.1095 & 0.1095 & 0.1096 & 0.1095 & 0.1097 & 0.1095 & 0.1096 & 0.1096 \\
\hline 0.2 & 0.2098 & 0.2098 & 0.2099 & 0.2098 & 0.2100 & 0.2098 & 0.2098 & 0.2098 \\
\hline 0.3 & 0.2919 & 0.2919 & 0.2920 & 0.2918 & 0.2921 & 0.2918 & 0.2919 & 0.2919 \\
\hline 0.4 & 0.3479 & 0.3479 & 0.3480 & 0.3479 & 0.3482 & 0.3479 & 0.3480 & 0.3480 \\
\hline 0.5 & 0.3716 & 0.3716 & 0.3716 & 0.3715 & 0.3719 & 0.3715 & 0.3716 & 0.3716 \\
\hline 0.6 & 0.3591 & 0.3590 & 0.3592 & 0.3590 & 0.3593 & 0.3590 & 0.3591 & 0.3591 \\
\hline 0.7 & 0.3099 & 0.3099 & 0.3101 & 0.3098 & 0.3102 & 0.3099 & 0.3099 & 0.3100 \\
\hline 0.8 & 0.2278 & 0.2278 & 0.2280 & 0.2278 & 0.2281 & 0.2278 & 0.2278 & 0.2279 \\
\hline 0.9 & 0.1207 & 0.1207 & 0.1208 & 0.1207 & 0.1208 & 0.1207 & 0.1207 & 0.1207 \\
\hline
\end{tabular}


TABLE II

ERROR ANALYSIS COMPARISON OF RESULTS AT $T=0.1$ FOR $\lambda=1, \Delta T=0.00001, H=0.0125$.

\begin{tabular}{ccccccc}
\hline$X$ & CHCM-L & CHCM-C & {$[12]$} & {$[13]$} & {$[14]$} & {$[15]$} \\
\hline 0.1 & $0.0000 \mathrm{E}+00$ & $-7.0000 \mathrm{E}-05$ & $2.0000 \mathrm{E}-05$ & $-1.1000 \mathrm{E}-04$ & $-1.0000 \mathrm{E}-05$ & $0.0000 \mathrm{E}+00$ \\
0.2 & $1.0000 \mathrm{E}-05$ & $-1.0000 \mathrm{E}-04$ & $4.0000 \mathrm{E}-05$ & $-1.9000 \mathrm{E}-04$ & $-2.0000 \mathrm{E}-05$ & $-1.0000 \mathrm{E}-05$ \\
0.3 & $0.0000 \mathrm{E}+00$ & $-1.6000 \mathrm{E}-04$ & $6.0000 \mathrm{E}-05$ & $-2.3000 \mathrm{E}-04$ & $-3.0000 \mathrm{E}-05$ & $-1.0000 \mathrm{E}-05$ \\
0.4 & $1.0000 \mathrm{E}-05$ & $-1.9000 \mathrm{E}-04$ & $7.0000 \mathrm{E}-05$ & $-2.6000 \mathrm{E}-04$ & $-4.0000 \mathrm{E}-05$ & $-1.0000 \mathrm{E}-05$ \\
0.5 & $0.0000 \mathrm{E}+00$ & $-7.0000 \mathrm{E}-05$ & $9.0000 \mathrm{E}-05$ & $-2.7000 \mathrm{E}-04$ & $-5.0000 \mathrm{E}-05$ & $0.0000 \mathrm{E}+00$ \\
0.6 & $1.0000 \mathrm{E}-05$ & $-2.6000 \mathrm{E}-04$ & $9.0000 \mathrm{E}-05$ & $-2.7000 \mathrm{E}-04$ & $-5.0000 \mathrm{E}-05$ & $1.0000 \mathrm{E}-05$ \\
0.7 & $1.0000 \mathrm{E}-05$ & $-2.0000 \mathrm{E}-04$ & $8.0000 \mathrm{E}-05$ & $-2.6000 \mathrm{E}-04$ & $-4.0000 \mathrm{E}-05$ & $2.0000 \mathrm{E}-05$ \\
0.8 & $0.0000 \mathrm{E}+00$ & $-2.7000 \mathrm{E}-04$ & $6.0000 \mathrm{E}-05$ & $-2.3000 \mathrm{E}-04$ & $-4.0000 \mathrm{E}-05$ & $2.0000 \mathrm{E}-05$ \\
0.9 & $0.0000 \mathrm{E}+00$ & $-1.0000 \mathrm{E}-04$ & $4.0000 \mathrm{E}-05$ & $-1.4000 \mathrm{E}-04$ & $-2.0000 \mathrm{E}-05$ & $2.0000 \mathrm{E}-05$ \\
\hline
\end{tabular}

TABLE III

COMPARISON OF RESULTS FOR $\Delta T=0.0001, H=0.0125, V=0.01$.

\begin{tabular}{lllllcc}
\hline & & Exact & OCFE-L & OCFE-C & {$[14]$} & {$[15]$} \\
\hline \multirow{4}{*}{0.25} & 0.40000 & 0.34191 & 0.34191 & 0.34190 & 0.34193 & 0.34190 \\
& 0.60000 & 0.26896 & 0.26896 & 0.26894 & 0.26898 & 0.26890 \\
& 0.80000 & 0.22148 & 0.22148 & 0.22147 & 0.22149 & 0.22150 \\
& 1.00000 & 0.18819 & 0.18819 & 0.18817 & 0.18820 & 0.18820 \\
& 3.00000 & 0.07511 & 0.07511 & 0.07509 & 0.07512 & 0.07510 \\
\hline \multirow{3}{*}{0.5} & 0.40000 & 0.66071 & 0.66071 & 0.66068 & 0.66076 & 0.66070 \\
& 0.60000 & 0.52942 & 0.52942 & 0.52940 & 0.52945 & 0.52940 \\
& 0.80000 & 0.43914 & 0.43914 & 0.43912 & 0.43916 & 0.43910 \\
& 1.00000 & 0.37442 & 0.37442 & 0.37440 & 0.37443 & 0.37440 \\
& 3.00000 & 0.15018 & 0.15018 & 0.15017 & 0.15018 & 0.15020 \\
\hline \multirow{3}{*}{0.75} & 0.40000 & 0.91026 & 0.91027 & 0.91021 & 0.91046 & 0.91030 \\
& 0.60000 & 0.76724 & 0.76724 & 0.76720 & 0.76733 & 0.76720 \\
& 0.80000 & 0.64740 & 0.64740 & 0.64738 & 0.64744 & 0.64740 \\
& 1.00000 & 0.55605 & 0.55605 & 0.55602 & 0.55608 & 0.55600 \\
& 3.00000 & 0.22481 & 0.22481 & 0.22479 & 0.22484 & 0.22480 \\
\hline
\end{tabular}

TABLE IV

COMPARISON OF RESULTS FOR $N=0.004,0.005$ AT DIFFERENT $X$ AND $T$ WITH $\Delta T=0.001, H=0.0125$.

\begin{tabular}{cccccccc}
\hline & \multicolumn{3}{c}{$v=0.004$} \\
\hline$X$ & $T$ & Exact & OCFE-C & OCFE-L & Exact & OCFE-C & OCFE-L \\
\hline 0.25 & 1 & 0.18889 & 0.18888 & 0.18887 & 0.18879 & 0.18877 & 0.18875 \\
& 5 & 0.04697 & 0.04696 & 0.04695 & 0.04696 & 0.04695 & 0.04696 \\
& 10 & 0.02421 & 0.02420 & 0.02419 & 0.02422 & 0.02421 & 0.02420 \\
& 15 & 0.01631 & 0.01620 & 0.01630 & 0.01631 & 0.01631 & 0.01631 \\
\hline 0.5 & 1 & 0.37596 & 0.37595 & 0.37594 & 0.37572 & 0.37570 & 0.37569 \\
& 5 & 0.09393 & 0.09393 & 0.09393 & 0.09392 & 0.09393 & 0.09393 \\
& 10 & 0.04843 & 0.04843 & 0.04843 & 0.04842 & 0.04841 & 0.04841 \\
& 15 & 0.03259 & 0.03259 & 0.03259 & 0.03244 & 0.03244 & 0.03244 \\
\hline 0.75 & 1 & 0.55881 & 0.55850 & 0.55880 & 0.55838 & 0.55836 & 0.55836 \\
& 5 & 0.14089 & 0.14089 & 0.14089 & 0.14083 & 0.14083 & 0.14083 \\
& 10 & 0.07220 & 0.07210 & 0.07210 & 0.07113 & 0.07112 & 0.07112 \\
& 15 & 0.04677 & 0.04678 & 0.04678 & 0.04413 & 0.04413 & 0.04414 \\
\hline
\end{tabular}

